\title{
Leveraging Social Media to Understand Public Perceptions toward Micromobility Policies: The Dallas Scooter Ban Case
}

\author{
Javad J. C. Aman ${ }^{1}$ (D), Janille Smith-Colin ${ }^{1}$ (D) \\ ${ }^{1}$ Civil and Environmental Engineering, Southern Methodist University, Dallas, TX, United States of America \\ Keywords: scooter, text mining, topic modeling, social media, micromobility, public policy \\ 10.32866/001c.21328
}

Findings

In this study, comments made on social media by City of Dallas residents were analyzed following a citywide scooter ban to reveal hidden attitudes towards micromobility. Using text mining techniques, results showed that the majority of residents were in fact against the ban. By applying term frequency-inverse document frequency (tf-idf), key terms mentioned by ban supporters and opponents were uncovered. Latent Dirichlet Allocation further showed that topics such as Bike, Public Safety, Authorities, Cars, and Feelings were discussed most frequently within resident comments. Findings demonstrate the potential for social media to clarify public perceptions towards public services such as micromobility.

\section{Questions}

Scooters were intended to offer a convenient and affordable last-mile/firstmile alternative, thus helping to shrink transit deserts (Aman and Smith-Colin 2020). However, in September 2020, two years after the City of Dallas implemented the Dockless Mobility pilot program, the City Department of Transportation abruptly halted the scooter program resulting in the removal of thousands of scooter vehicles from streets. Officials cited public safety concerns and "complaints from residents" as reasons for the scooter ban (Steele and Keomoungkhoun 2020). As news of the scooter ban was broadcast by local media, residents reacted in real-time via social media to the decision. Some residents posted comments expressing opinions about the scooter ban to the local Instagram pages, while others reacted to the news via Twitter. Comments offered support for the ban; others criticized the ban and a few offered solutions to the micromobility challenges facing the City. In this study, comments made by residents about the scooter ban on social media were extracted, and text mining techniques applied to answer the following research questions:

- What were the opinions expressed by residents about the ban?

- What latent topics did residents discuss in their comments?

\section{Methods}

More than 750 textual comments and tweets related to the scooter ban were identified and extracted for analysis via web crawlers. Comments were short, with an average word count of $11(S D=13)$. Data preprocessing included tokenization, lowercasing, non-informative character removal, typo correction, and lemmatization. Opinions expressed in the comments were then manually coded by one researcher and corroborated by a second researcher as either 


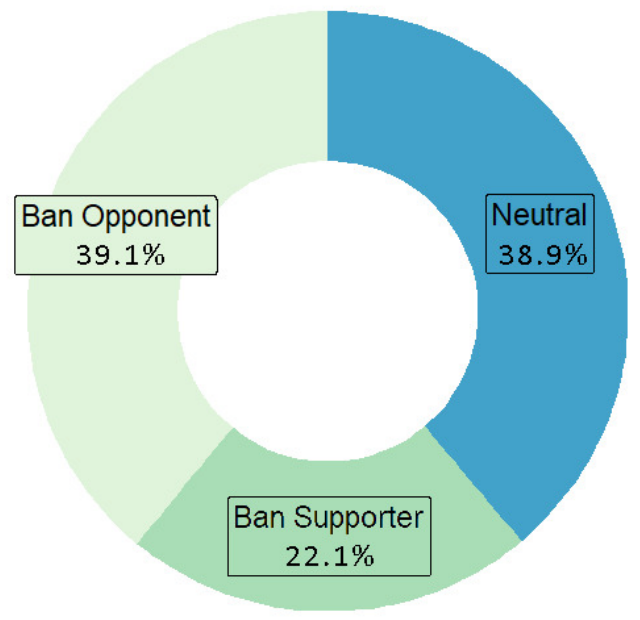

Figure 1- Opinions toward the scooter ban

ban supporter, ban opponent, or neutral. Comments expressing aversion to or dissatisfaction with the ban were labeled opponents, and those expressing satisfaction with or support for the ban were labeled supporters. Comments expressing an unclear position towards the ban (e.g., comments with only emojis, sarcasm, or vague language), were labeled neutral.

To identify keywords used in supporter and opponent comments, tf-idf weights were calculated. Tf-idf is a statistical approach that not only considers the frequency of term occurrence but takes into consideration the importance of the term within the text. Latent Dirichlet Allocation, an unsupervised topic modeling method for document classification, was also applied to identify hidden themes within the text (Blei, Ng, and Jordan 2003). Beginning with 20 topics, an iterative process of reducing the number of topics was undertaken to achieve an optimal number of topics.

\section{Findings}

Figure 1 shows that $38.9 \%$ of comments were neutral; that is residents expressed neither opinions of support nor opposition to the ban. Among individuals who expressed an opinion regarding the ban, $39.1 \%$ were opponents of the ban, and $22.1 \%$ were supporters of the ban.

Figure 2 shows common and important words mentioned in the comments. Ban supporters used words such as "pedestrian," "hit" and"dangerous" highlighting concerns about safety. On the other hand, ban opponents used terms like "alternative," "car," and "job" suggesting a focus on the mobility and accessibility benefits offered by scooters as a transportation alternative. 


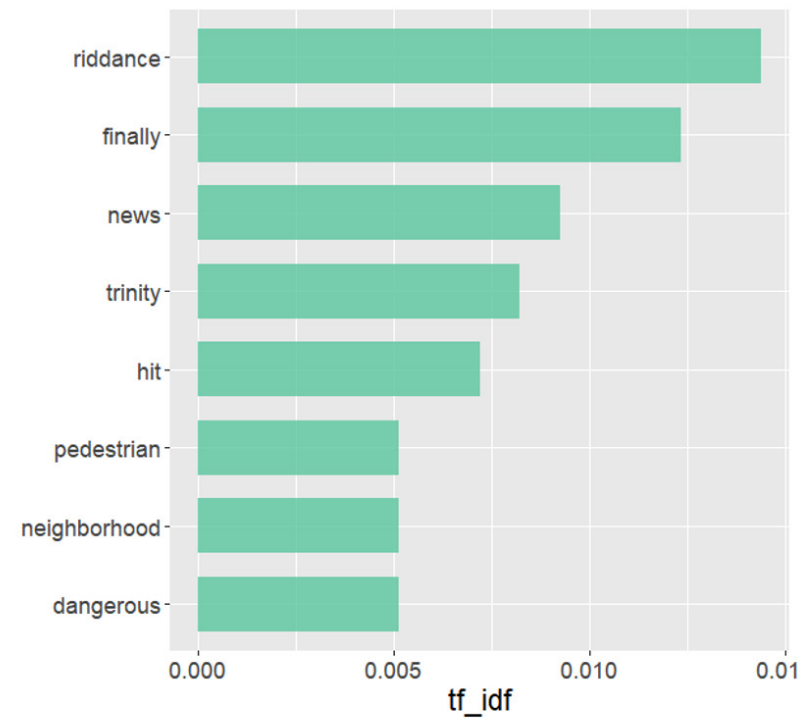

a- Ban supporters

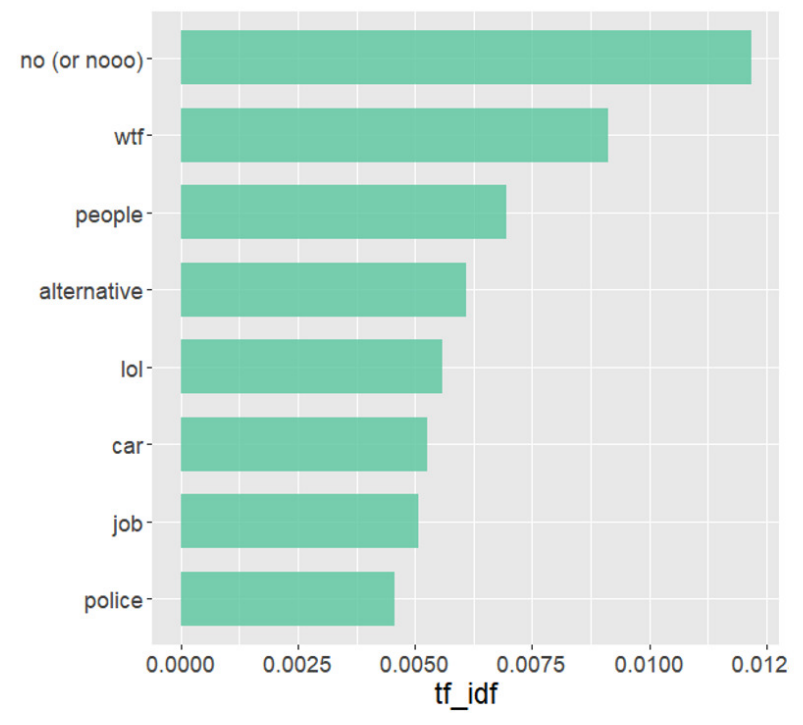

b- Ban opponents

Figure 2- tf-idf results

Table 1- Extracted topics

\begin{tabular}{lll}
\hline No. & Topic & Frequent Terms \\
\hline 1 & Bike & Bike, City, WTF, Option, Public, Return, Decision \\
2 & Feelings & Sad, News, Bye, Lame, Wow, Damn, Fun \\
3 & Authorities & City, Decision, Deep Ellum, Council, People, Mayor \\
4 & Cars & Car, Driver, Riddance, Miss, Fall, Road, Sidewalk \\
5 & Public Safety & Crime, Dangerous, Neighborhood, Park, walk, experience, \\
\hline
\end{tabular}

Five topics or collections of words were identified, using topic modeling, each representing themes discussed throughout the data set (i.e., comments). Table 1 presents the final topics extracted from the topic modeling, including Bike, Feelings, Authorities, Cars, and Public Safety, and the most frequent terms related to each topic.

Bike: Within the Bike comments, residents often compared the scooter ban and related events to past experiences with dockless bikes within the City of Dallas, lamenting the loss of shared bikes, recounting memories, and expressing a desire to have bikes return to the streets as a transportation alternative. Shared bikes were once pervasive, but in June 2018, three major micromobility companies left the city, discontinuing their bike-share programs, following a City Council decision to tighten regulations (Dent 2019).

Feelings: In this topic, individuals expressed opinions related to the scooter ban, such as "about time," "bye scooters," and "sad/good news." Comments were generally brief and not overly informative. 
Authorities: In this topic, comments often addressed city officials directly, mostly via Twitter, to express opinions about the scooter ban. These comments mostly followed a tweet made by the Mayor of Dallas criticizing the scooter ban for being enacted without City Council action. Deep Ellum, a scooter hotspot and an entertainment neighborhood known for its vibrant environment was one of the most discussed subtopics in these comments.

Cars: In this topic, residents mostly expressed the belief that removing scooters would further empower the "car culture" and contribute to the belief that roads are car territory. These opinions seemed to be mostly expressed by scooter riders. Yet others, believed to be car drivers, also expressed support for the ban sighting the conflict that scooters create with cars.

Public Safety: In this topic, residents generally debated the role that scooters play in public safety - are they a threat or not? Subtopics were varied and included things such as the use of scooters as getaway vehicles following a crime, inappropriate parking, and riding under the influence.

Previous studies have highlighted the importance of user generated content in understanding micromobility services (Aman and Smith-Colin 2021; Feng et al. 2020). Our findings particularly demonstrate the value of social media comments for clarifying public perceptions towards public policy actions, including individual feelings towards services and factors influencing opinions. In an environment where perceptions towards micromobility remain unclear and micromobility opponents, in this case ban supporters, often have the loudest voice, text analysis of social media comments can provide a mechanism to rapidly uncover hidden attitudes. It is important to consider, however, that these findings reflect the voices of those who participate in social media. Moreover, as only 750 comments and tweets were collected, additional public engagement may ultimately be necessary to gain an unbiased understanding of public opinion. 


\section{REFERENCES}

Aman, J. J. C., and J. Smith-Colin. 2020. "Transit Deserts: Equity Analysis of Public Transit Accessibility." Journal of Transport Geography 89 (December): 102869. https://doi.org/10.1016/ j.jtrangeo.2020.102869.

- - - 2021. "Application of Text Mining Techniques on User-Generated Reviews to Understand Scooter Rider Satisfaction.” Presented at the Transportation Research Board Annual Meeting, TRB Session 1248 - Emerging Research in Bikeshare and Micro-Mobility, Presentation Number: TRBAM-21-03263.

Blei, D. M., A. Y. Ng, and M. I. Jordan. 2003. "Latent Dirichlet Allocation.” Journal of Machine Learning Research 3 (January): 993-1022.

Dent, M. 2019. “Can Car-Crazy Dallas Learn to Love Bikes?,” October 16, 2019.

Feng, Y., D. Zhong, P. Sun, W. Zheng, Q. Cao, X. Luo, and Z. Lu. 2020. "Micromobility in Smart Cities: A Closer Look at Shared Dockless E-Scooters via Big Social Data.” arXiv preprint arXiv:2010.15203.

Steele, T., and N. Keomoungkhoun. 2020. "Electric Scooters Must Be Taken off Dallas' Streets by the End of the Week, City Says.” The Dallas Morning News, 2020. 